\title{
A FIBERING THEOREM FOR TOPOLOGICAL SEMIGROUPS
}

\author{
PAUL S. MOSTERT ${ }^{1}$
}

The theorem of this paper has appeared under stronger hypotheses and sometimes with weaker conclusions a number of times $[1 ; 2 ; 3]$, and was known to Koch (in approximately the form of [2]) in 1959 (unpublished). Since it is a useful tool in the study of topological semigroups, and the proof here is simpler, and the theorem stronger than those mentioned, it seems advisable to have it in the literature.

LEMMA 1. Let $X$ be a compact space and $G$ a compact group of homeomorphisms of $X$. If $X$ contains a fixed point under $G$, and $X / G$ is connected, then $X$ is connected.

Proof. Let $X=A \cup B$ where $A$ and $B$ are compact, open subsets of $X$. Suppose $p \in B$ is a fixed point. Now $X=(B \backslash G(A)) \cup G(A)$ determines a separation in $X / G$. Hence, either $A$ is empty or $B \subset G(A)$. But the latter implies $p \in A$.

Definitions. Let $X$ be a Hausdorff space, and $G$ a compact group of homeomorphisms of $X$, and $p \in X$. A semi-slice at $p$ is a closed set $T \subset X$ such that $p \in T$, and if $x \in T, h \in G$, then $h(x) \in T$ if and only if $h \in G_{p}$.

If, further, there is a local cross section to the orbits of $G_{p}$ in $G$, then $T$ is a slice $[4 ;$ p. 105] and $G(T)$ is a bundle over $G(p)$ with fibre $T$ and group $G_{p}$. This can easily be generalized to the following with essentially identical proof.

LemMa 2. Let $X$ be a Hausdorff space, $G$ a compact group of homeomorphisms of $X$, and $T$ a semi-slice at $p$. If $F \subset G(p)$, and $F^{*}=\{g \in G: g(p) \in F\}$, then $F^{*}(T)$ is a bundle over $F$ if there is a local cross section in $F^{*}$ to the orbits of $G_{p}$.

In particular, then, since finite polyhedra and a homotopy of such in $G(p)$ can be "lifted" into $G$ [5], we have

LEMмA 3. Let $X$ be a Hausdorff space and $G$ a compact group of homeomorphisms of $X$. If there is a semi-slice $T$ at $p \in X$, then $G(T)$ is a fibre space in the sense of Serre with base space $G(p)$ and group $G_{p}$.

THEOREM. Let $S$ be a compact connected semigroup with identity 1, and denote by $H$ the maximal subgroup of the identity and $K$ the kernel. If $K$ is contained in an orbit of $H$ under left multiplication then:

(i) $K$ is a group with identity $e$,

(ii) the map $\pi: H \rightarrow K$ defined by $\pi(h)=e h$ is a homomorphism onto,

Received by the editors January 27, 1962.

1 This work was supported by the National Science Foundation. 
(iii) $T=\{x \in S:$ ex $=e\}$ is a compact connected subsemigroup with zero and identity.

(iv) the maximal subgroup $H_{e}$ of $T$ is $T \widehat{\cap} H$,

(v) $S$ is a fibre space in the sense of Serre with base $K$ and fibres homeomorphic to $T$, and is a fibre bundle (with group $H_{e}$ ) if the cosets of $H_{0}$ in $H$ admit a local cross section.

Proof. It is well known that if $K$ is contained in an orbit of $H$, then it is a group and the mapping $\pi$ is a homomorphism onto [ 3 ]. Further, $T$ is clearly a closed subsemigroup containing 1 , and $e$ is a zero. That $H_{e}=T \cap H$ is also clear. If $x \in S$, then $e x \in K$ and since $e H=K$, there is an element $h \in H$ such that $e h=e x$. Hence, $x h^{-1} \in T$. That is, $T$ meets each orbit. Now if $x \in T$, and $x h \in T$, it follows that $e h=e x h=e$ so that $h \in T$. Thus, $T$ is a semi-slice. Hence, $T / H_{0}=S / H$, and by Lemma $1, T$ is connected. The result now follows from Lemma 3.

REMARK 1. If $K$ is finite-dimensional, it is known [5] that there is a local cross section to the orbits of $H_{a}$ in $H$.

REMARK 2. As one sees immediately from the proof, the connectedness of $S$ would follow if, instead of assuming $S$ connected, we assume $K$ and $S / H$ connected.

\section{BIBLIOGRAPHY}

1. K. H. Hofmann, Homogeneous locally compact groups with compact boundary, Habilitationsschrift, Tuebingen Universitaet, 1962.

2. R. P. Hunter and N. J. Rothman, Characters and cross sections for certain semigroups, Duke Math. J. 29 (1962), 347-366.

3. P. S. Mostert, and A. L. Shields, On the structure of semigroups on a compact manifold with boundary, Ann. of Math. (2) 65 (1957), 117-143.

4. A. Borel, Seminar on Transformation Groups, Annals of Mathematics Studies, no. 46, Princeton Univ. Press, Princeton, N. J., 1960.

5. P. S. Mostert, Sections in principal fibre spaces, Duke Math. J. 23 (1956), 5772.

TULANE UNIVERSITY 